\title{
Delocalization of Wannier-Stark ladders by phonons: tunneling and stretched polarons
}

\author{
Wei Zhang, ${ }^{a}$ Alexander O. Govorov, ${ }^{a, b}$ and Sergio E. Ulloa ${ }^{a}$ \\ ${ }^{a}$ Department of Physics and Astronomy, and Condensed Matter and Surface Sciences Program, Ohio University, \\ Athens, Ohio 45701-2979 \\ ${ }^{b}$ Institute of Semiconductor Physics, RAS, Siberian Branch, 630090 Novosibirsk, Russia
}

\begin{abstract}
We study the coherent dynamics of a Holstein polaron in strong electric fields. A detailed analytical and numerical analysis shows that even for small hopping constant and weak electronphonon interaction, polaron states can become delocalized if a resonance condition develops between the original Wannier-Stark states and the phonon modes, yielding both tunneling and 'stretched' polarons. The unusual stretched polarons are characterized by a phonon cloud that trails the electron, instead of accompanying it. In general, our novel approach allows us to show that the polaron spectrum has a complex nearly-fractal structure, due to the coherent coupling between states in the Cayley tree which describes the relevant Hilbert space. The eigenstates of a finite ladder are analyzed in terms of the observable tunneling and optical properties of the system.
\end{abstract}

The coupling of an electronic system with a coherent boson field has been a subject of great interest in recent years, thanks to the availability of high quality materials and intense photon sources. In condensed matter systems, however, a natural intrinsic field of bosonic nature is provided by the lattice vibrations. The coupling to phonons is typically considered to result in inelastic incoherent scattering for the electron, as the phonons are difficult to interrogate separately. This results in an electronic 'open' system which exists immersed in the assumed-incoherent phonon field. Although this description is appropriate for many situations, either because the electron-phonon coupling is weak and/or non-resonant, or if the system is at high temperatures, its validity is suspect if the interaction is effectively strong and the temperature is low.

Electronic transport in superlattices has yielded a number of interesting phenomena and concepts, including Bloch oscillations and Wannier-Stark (WS) ladders [1], as well as negative differential conductance [2] in high DC electric fields, dynamical localization [3], fractional WS ladders under DC and AC electric fields [甘, , and resonant magnetopolarons $[5$. Similarly, electron-phonon interactions under high electric fields have been investigated in some detail, including coupled Bloch-phonon and -plasmon oscillations [6], as well as phonon-assisted-hopping of an electron on a WS ladder [7]. A powerful variational treatment of inelastic but coherent quantum transport was presented in [8], while anomalies in transport properties under a resonant condition were studied in [9], and the optical absorption associated with the resonance of a WS ladder and confined optical phonons was studied in Refs. [10,11].

In this paper, we study the effects of coherent coupling of an electron to the phonon system present in its solid at low temperatures. We are specially interested in the effect of this coupling on the tunneling properties of an electron in an intense electric field region, like the situation achieved in semiconductor superlattices, for example [12]. Our description is non-perturbative for the resonant case with exponentially large Hilbert space, and as such we are able to elucidate the role of resonant phonon fields on the otherwise localized electron residing in a WS ladder, for both weak and strong coupling. Using a Holstein model for the electron-phonon coupling, and both analytical and numerical techniques, we demonstrate that the electron tends to become more extended for increasing coupling with phonons. Moreover, we show that resonant coupling results in total delocalization of the polaron for some of the states, and the consequent restoring of a 'miniband' structure in the system, even as the applied electric field remains strong. Other 'stretched polaron' states, however, are highly degenerate yet strongly localized on one end of a finite structure, with a phonon 'cloud' which is nearly detached from the electron (somewhat a precursor of the polaron dissociation described in [13]). We are able to show explicitly that the level spectrum in this regime has a nearly fractal structure with rather complex wave functions. On the other hand, when the system is away from the resonant condition, a deformed WS ladder results, with electronic wave functions which nevertheless remain localized. We finally analyze the tunneling and optical properties one could observe experimentally in such a system.

We consider a Holstein model [14], describing a single electron in a one-dimensional tight-binding lattice which interacts locally with dispersionless optical phonons. In addition, we consider that a strong constant electric field is applied which generates a WS ladder. The Hamiltonian is then

$$
\begin{aligned}
H_{0}= & \sum_{j}\left\{\varepsilon_{j} c_{j}^{+} c_{j}+t\left(c_{j}^{+} c_{j+1}+c_{j+1}^{+} c_{j}\right)\right\} \\
& +\omega \sum_{j} a_{j}^{+} a_{j}+\gamma \sum_{j} c_{j}^{+} c_{j}\left(a_{j}^{+}+a_{j}\right),
\end{aligned}
$$

where $t$ is the electron hopping constant, $\omega$ the phonon frequency, $\gamma$ the electron-phonon coupling constant, $\varepsilon_{j}=$ $-e d E j \equiv-j \Delta$ the site (or 'well') energy, $E$ the electric field, $d$ the lattice constant, and $\Delta$ the spacing between WS 'rungs' (we have set $\hbar=1$ ). It is known that well-localized WS states will form in the absence of electron-phonon 
interactions, with eigenvalues and eigenfunctions given by $\varepsilon_{j}$, and $\left|\phi_{j}\right\rangle=\sum_{i} J_{i-j}(2 t / e d E)|i\rangle$, where $J_{l}$ is the $l$-th order Bessel function [3]. Given the properties of $J_{l}$, the WS $j$-state is clearly localized with a characteristic length $2 t / e E$ around site $j$. It is helpful to introduce the WS creation and annihilation operators, $d_{j}=\sum_{i=-\infty}^{\infty} J_{i-j}(2 t / e d E) c_{i}$. It is easy to show that $\left\{d_{i}^{+}, d_{j}\right\}=\delta_{i j}$, and that the Hamiltonian can be rewritten in terms of $d_{j}, d_{j}^{+}$. In the case of strong electric field (or small hopping constant) $(2 t / \Delta \ll 1)$, the Hamiltonian can be simplified to

$$
\begin{aligned}
H= & \sum_{j}\left\{-\Delta j d_{j}^{+} d_{j}+\omega a_{j}^{+} a_{j}+\gamma d_{j}^{+} d_{j}\left(a_{j}^{+}+a_{j}\right)\right. \\
& \left.-\lambda\left(a_{j}+a_{j}^{+}-a_{j+1}^{+}-a_{j+1}\right)\left(d_{j}^{+} d_{j+1}+d_{j+1}^{+} d_{j}\right)\right\},
\end{aligned}
$$

where the effective hopping becomes $\lambda=\gamma t / \Delta$. Notice that phonon-assisted hopping develops between the WS states via the last term. Eq. (2) is similar to that in [10], but here, as the electron jumps it can not only emit (or absorb) a phonon on its current location, but also on the neighboring site. This more natural description results in a much larger relevant Hilbert space for this problem.

Consider the process of an electron jumping between WS states and creating or annihilating a phonon with amplitude $\lambda$, as per the last term in (2). The relevant Hilbert space can be described for $n=3$ via the basis: $|1 ; 000\rangle,|2 ; 010\rangle,|2 ; 100\rangle,|3 ; 011\rangle,|3 ; 101\rangle,|3 ; 020\rangle$, and $|3 ; 110\rangle$. Each state $\left|j ; m_{1}, m_{2}, \ldots\right\rangle$ is indexed by the electron position $j$, and $m_{k}$ refers to the number of phonons on site $k$. This construction yields a Hilbert space of dimension $N-1=2^{n}-1$, where $n$ is the total number of sites in the chain. It is interesting to note that the structure of this vector space is that of a Cayley tree where each $j$-state links to two at $j+1$, in general with non-symmetric weights in all branches [15]. A similar structure of the vector space was found in [8].

Under the resonance condition, i.e., $\Delta=\omega$, all the basis-states in the Hilbert space described above have the same energy, and one would expect that the off-diagonal matrix elements in (2) would break the degeneracy. One would expect that a 'miniband' of extended states might form, whenever the resonance condition is reached. For this case, Hamiltonian (2) in the above basis of $n=3$ takes the form

$$
H=\left(\begin{array}{ccccccc}
-\Delta & \lambda & -\lambda & 0 & 0 & 0 & 0 \\
\lambda & -\Delta & 0 & \lambda & 0 & -\sqrt{2} \lambda & 0 \\
-\lambda & 0 & -\Delta & 0 & \lambda & 0 & -\lambda \\
0 & \lambda & 0 & -\Delta & 0 & 0 & 0 \\
0 & 0 & \lambda & 0 & -\Delta & 0 & 0 \\
0 & -\sqrt{2} \lambda & 0 & 0 & 0 & -\Delta & 0 \\
0 & 0 & -\lambda & 0 & 0 & 0 & -\Delta
\end{array}\right),
$$

with ever larger blocks for larger $n$ values. Notice that there are elements with somewhat unusual values, $-\sqrt{2} \lambda$, associated with the higher number of phonons in state $|3 ; 020\rangle$. A similar structure is seen for all values of $n$, and as these terms appear only sporadically, they have only a small influence on the overall level structure. In particular, one expects that there would be little change in the physics in the limit of large $N$, if we replace $-\sqrt{2} \lambda$ terms with $-\lambda$. This assumption is in fact confirmed explicitly by numerical calculations. Moreover, this 'symmetrized' Hamiltonian $H_{\text {sym }}$ allows one to better analyze the structure of the solutions of $H$, as we show below, while exhibiting some of the same generic features and fully self-similar properties.

Symmetrized Hamiltonian. By using the block decomposition of the $\operatorname{determinant,} \operatorname{det}\left(\begin{array}{cc}A & B \\ C & D\end{array}\right)=\operatorname{det}(D) \operatorname{det}(A-$ $B D^{-1} C$ ), one can find that the eigenvalues of $H_{\text {sym }}$ are determined by the equation $\varepsilon_{0}^{N / 2} \varepsilon_{1}^{N / 4} \varepsilon_{2}^{N / 8} \cdots \varepsilon_{n-1}^{1}=0$, where $\varepsilon_{0}=\varepsilon, \varepsilon$ is the energy eigenvalue, and $\varepsilon_{k+1}=\varepsilon-2 \lambda^{2} / \varepsilon_{k}$. Here we have made a constant energy shift of $\Delta$. One can then write $\varepsilon_{k}$ as a continuous fraction in $k$ steps

$$
\varepsilon_{k}=\varepsilon-\frac{2 \lambda^{2}}{\varepsilon-\frac{2 \lambda^{2}}{\varepsilon-\dot{亠}}} .
$$

and the eigenvalues of $H_{\text {sym }}$ are given by [11] [16]

$$
\varepsilon_{k, l}=2 \sqrt{2} \lambda \cos \left(\frac{l \pi}{k+1}\right),
$$

where $k=1,2, \ldots, n ; l=1, \ldots, k$. The limiting bandwidth of the spectrum is $4 \sqrt{2} \lambda$.

Using $H_{\text {sym }}$, one can arrive at an estimate for the degeneracies for the first few eigenvalues in the limit of large $N$, given by the equation $\varepsilon^{3 N / 8}\left(\varepsilon^{2}-2 \lambda^{2}\right)^{N / 8}\left(\varepsilon^{2}-4 \lambda^{2}\right)^{N / 8}=0$. One then has eigenvalues $\varepsilon=0$ with degeneracy $3 N / 8$, 
$\varepsilon= \pm \sqrt{2} \lambda$ with degeneracy $N / 8$, and $\varepsilon= \pm 2 \lambda$ with degeneracy $N / 8$, in agreement with our explicit calculations for finite $N$. Notice that increasing lattice or chain size produces both new energy eigenvalues within the limiting band and additional states which augment the degeneracy of some of the old eigenvalues. The overall structure of the spectrum remains the same and all the eigenvalues of the shorter chain are also in the spectrum of the longer one [17].

Figure 1 shows typical numerical results for the eigenvalues of the physical Hamiltonian $H$ in Eq. (2), while the inset shows the results for the symmetrized version $H_{\text {sym }}$ (both for $n=8$ ). Our analytical results match exactly those shown in the inset, which in turn mimic well the results for $H$ in the main panel, although small differences appear due to the sporadic $-\sqrt{2} \lambda$ terms. The self-similar structure of the spectrum in the inset is the manifestation of the full self-similarity of the Hamiltonian $H_{\text {sym }}$ (while it is only approximate for $H$ ). One can see that the original highly degenerate Hilbert basis is split into a semi-continuous 'miniband' by the off-diagonal elements in $H$ (and which for $H_{\text {sym }}$ yields Eq. 5 for large $N$ ). Notice, however, that there are large residual degeneracies for the eigenvalues of $H$ in the center of the miniband. The right panel in Fig. 1 shows the density of states of the $H$ system, exhibiting also an approximate self-similarity.

The 'electronic wave functions' for various sample eigenstates are shown in Fig. $2, P_{\varepsilon}(j)=\sum_{\{m\}}\left|C_{j,\{m\}}^{\varepsilon}\right|^{2}$, where the $\varepsilon$-eigenstate is given in terms of the basis above by $\left|\Psi_{\varepsilon}\right\rangle=\sum_{j,\{m\}} C_{j,\{m\}}^{\varepsilon}|j ;\{m\}\rangle$. One can observe that the states in regions of high degeneracy, $\varepsilon=0$ and $\varepsilon / \lambda \approx-1.6$ (where states $(\mathrm{A})$ and $(\mathrm{C})$ are indicated), are localized around the right end of the structure, $j \approx 8$. However, non-degenerate states in the band, such as the one labeled (B), result to be fully extended over the structure.

Notice that in this inherently coupled electron-phonon system, the calculated eigenstates are a coherent mixture of electron and phonons on different sites. The amplitudes in Fig. 2 have been projected over all phonon components. Similarly, in order to illustrate the physics of these states better, one can exhibit the average phonon number for various states in Fig. 3. One can see clearly that all states at the degenerate center of the band have many more phonons $(\simeq 7)$ than those non-degenerate states residing higher in the band. It appears, in fact, that when the electron interacts with many phonons, it localizes, as one would perhaps intuitively expect for polaron self-trapping states, while states with fewer phonons are less localized and 'propagate' better. One can provide an estimate for the average number of phonons for extended states by the following argument: The average phonon number is given by $\langle N\rangle=\sum_{i} P_{i} N_{i}$, where $P_{i}$ is the probability for an electron to be at site $i$, and $N_{i}$ is the number of phonons in this state. For an extended state, $P_{i} \simeq 1 / n$, where $n$ is the number of sites. A state with an electron at site $i$ can be obtained by the electron hopping $i-1$ steps from the first site, while emitting $N_{i}=i-1$ phonons in the process. We then obtain that the average number of phonons for an extended states is $\langle N\rangle \simeq \sum_{i}(i-1) / n=(n-1) / 2$. This result matches well the numerical results, where the most extended states have $\langle N\rangle \simeq 3.5$ in Fig. 3, such as the state labeled (B).

One can also inspect the spatial distribution of phonons for each state, as in the inset of Fig. 2, which shows the corresponding phonon distribution for several eigenstates, $N_{\varepsilon}(j)=\sum_{l,\left\{m_{j}\right\}}\left|C_{l,\left\{m_{j}\right\}}^{\varepsilon}\right|^{2} m_{j}$, indicating how the phonons distribute over the chain. The degenerate states, such as (A) and (C), with the electron localized to the right of the structure, have nearly one phonon per site throughout the chain, indicating that these polarons have a 'stretched' phonon cloud away from the charge, seemingly in anticipation of the total polaron dissociation that other authors have described for extended chains under high electric fields [13]. In contrast, the most delocalized states (such as (B) in Fig. 2) show a phonon amplitude much less than unity throughout, yielding an overall low phonon count, and a cloud that in general 'accompanies' the charge.

Interband optical absorption. The spectrum of optical absorption associated with transitions between valence and conduction bands provides information about the density of states and the states' charge distribution. The absorption intensity $K$ is calculated from $K=\frac{2 \pi}{\hbar}\left|W_{\nu}\right|^{2} \delta\left(\varepsilon_{\nu}+E_{g}-\omega\right)$, where $E_{g}$ is the valence and conduction band gap, $\nu$ refers to the various polaron states, and $W_{\nu}$ is the transition matrix between valence band and conduction band states. Under the strong field present in the system, the hole is localized the most because of its large effective mass. Thus we ignore its negligible tunneling and coupling to phonons and assume it localized at site 1. Correspondingly, there is conservation of phonon number and $\left|W_{\nu}\right|^{2} \propto P_{\nu}(1)[18]$. In Fig. 4(a) we show the absorption intensity vs. phonon frequency. Once again, we see that the non-degenerate states, $\varepsilon / \lambda \simeq \pm 0.6$, have the largest amplitude at site 1 and will then contribute the most to the absorption spectrum. In contrast, all highly degenerate states at $\varepsilon=0$ are strongly localized at the right end of the chain and make a vanishing contribution to $K$. Such strong modulation of $K$ would be easily accessible to experiments.

Transport properties. As described above, tunneling experiments under strong electric fields are possible in semiconductor superlattices and other systems, and it is of interest to investigate the role of the resonance condition. The transition probability $P$ can be calculated in a $S$-matrix formalism. $P=|T|^{2}$ and $T=\left\langle\varepsilon_{f}, R|S| \varepsilon_{i}, L\right\rangle$. Here $R$ and $L$ refer to the right and left leads. In the wide band limit, $T \propto \Gamma \int d t_{1} d t_{2} e^{i\left(\varepsilon_{f} t_{2}-\varepsilon_{i} t_{1}\right)}\left\langle n\left|G^{R}\left(t_{2}-t_{1}\right)\right| 1\right\rangle=\sum_{\nu} \Gamma\langle n \mid \nu\rangle\langle\nu \mid 1\rangle \delta\left(\varepsilon_{f}-\varepsilon_{i}\right)$, 
where $\Gamma$ describes the electron interaction with contacts, and $G^{R}$ is the retarded Green function connecting both ends of the structure (the site $j=1$ and $j=n$ ) [19]. We use $D(\nu)=|\langle n \mid \nu\rangle\langle\nu \mid 1\rangle|^{2}=P_{\nu}(1) P_{\nu}(n)$ to describe the relative contribution of various eigenstates $\nu$ to the electronic transport, as the tunneling probability is proportional to the density of states in the leads and $D(\nu)[19$. Figure 4(b) shows the quantity $D(\varepsilon)$. We can see that all the states at the center of the band contribute zero to the transport amplitude through the chain, and that this behavior is exhibited by all the high peaks in the DOS, clearly consistent with their spatially localized-charge nature. On the other hand, $D$ shows large values for extended states, confirming in fact that the non-degenerate states in the miniband are extended. The variations shown in $D$ would then be reflected in strong amplitude modulations within each of the phonon replicas in tunneling experiments [20], whenever the resonance condition is reached. It is also clear that despite the fact that $K$ and $D$ correspond to completely different physical quantities, their energy behavior is surprisingly similar. This arises because of the large electric field asymmetry, resulting in no states with mostly/only large electronic amplitude in the first site (i.e., localized towards the left of the chain). Once a state is extended and has non-vanishing amplitudes at site 1 , it also does at site $n$.

So far we have mainly described the resonant case. When the system is away from the resonant condition, a deformed lattice with substructure is formed. We are also able to study the system beyond small hopping and weak coupling regime by mapping it via the Lang-Firsov canonical transformation to the case we have studied, with renormalized hopping and coupling constants. In this approach, we can provide additional physical insights into some interesting results found in [8]. We will report the details elsewhere [17].

Although our model is 1D [12], we expect the qualitative features and main conclusions to be valid for semiconductor superlattices (SLs) where electron-optical-phonon coupling is highly anisotropic. In that case, it would be possible to neglect the in-plane scattering of electrons by phonons, while the strong interaction assists in the electron hopping. In fact, for SLs with low in-plane disorder, the transitions between different layers involve states near the bottom of the 2D electron subbands and phonons with small in-plane momenta. Hence, the system would exhibit quasi-1D behavior, and in-plane scatterings are a small correction. Similarly, we could adapt our model to the description of polarons in polymer chains, such as those described in Ref. [13], although there the electron-phonon interaction is of different strength than in solid SLs, and tunneling experiments are rather more challenging.

We have studied the coherent dynamics of Holstein polarons in a strong electric field. It is found that with the help of phonons, each WS rung develops into a 'miniband' under the resonance condition. This miniband shows a nearly self-similar structure, which is inherited from the full self-similarity of the Hamiltonian $H_{\text {sym }}$ for a symmetric Cayley tree. Although the phonons can help the electron jump from one WS state to another, the phonons can also prevent the electron from propagating. If too many phonons are involved, this results in highly degenerate states with the electron localized at one end of the structure while the phonons are pulled away from it (the 'stretched' polaron). The miniband structure and its modulations in density of states and other characteristics are manifested in both transport and optical properties of the system, which we anticipate can be observed in experiments at low temperatures 12.

\section{ACKNOWLEDGMENTS}

This work was supported in part by US DOE No. DE-FG02-91ER45334.

[1] C. Waschke et al., Phys. Rev. Lett. 70, 3319 (1993);

E. E. Mendez, F. Agullo-Rueda, and J. M. Hong, Phys. Rev. Lett. 60, 2426 (1988);

E. E. Mendez and G. Bastard, Phys. Today 46, 34 (1993).

[2] R. Tsu and G. H. Dohler, Phys. Rev. B 12, 680 (1975).

[3] D. H. Dunlap and V. M. Kenkre, Phys. Rev. B. 34, 3625 (1986); Phys. Lett. A 127, 438 (1998); W. Zhang and X.-G. Zhao, Physica E 9, 667 (2001).

[4] X.-G. Zhao, R. Jahnke, and Q. Niu, Phys. Lett. A 202, 297 (1995).

[5] L.I. Korovin and S.T. Pavlov, Sov. Phys. JETP 26, 979 (1968);

Das Sarma and A. Madhukar, Phys. Rev. B 22, 2823(1980);

F.M. Peeters and J.T. Devreese, Phys. Rev. B 31, 3689(1985).

[6] A. W. Ghosh, L. Jonsson, and J. W. Wilkins, Phys. Rev. Lett. 85, 1084 (2000);

T. Dekorsy, et al., Phys. Rev. Lett. 85, 1080(2000). 
[7] D. Emin and C. F. Hart, Phys. Rev. B 36, 2530 (1987).

[8] J. Bonca, S. A. Trugman, Phys. Rev. Lett. 79, 4874(1997).

[9] V. V. Bryxin and Y. A. Firsov, Solid State Commun. 10, 471(1972);

V. L. Gurevich, V. B. Pevzner, and G. Iafrate, Phys. Rev. Lett. 75, 1352 (1995).

[10] A. O. Govorov and M. V. Entin, Sov. Phys. JETP 77, 819 (1993).

[11] A. O. Govorov, Solid State Commun 92, 977(1994).

[12] We should emphasize that our 1D formalism is strictly valid for a stack of quantum dots, like those achieved recently [see e.g. P.M. Petroff, A. Lorke and A. Imamoglou, Phys. Today 54, 56 (2001)], or for a superlattice under a normal magnetic field (since the Landau level quantization reduces the symmetry of the problem).

[13] S. V. Rakhmanova and E. M. Conwell, Appl. Phys. Lett. 75, 1518(1999).

[14] T. Holstein, Ann. Phys. (N.Y.) 8, 343(1959).

[15] For references on Cayley tree properties, see for example K. Kundu and B. C. Gupta, cond-mat/9705150.

[16] H. S. Wall, Analytic theory of continued fractions, D. Van Nostrand Co., New York, 1948.

[17] W. Zhang, A. O. Govorov, and S. E. Ulloa, unpublished.

[18] For a long chain, there are equivalent "site 1" positions, as each WS rung develops into its own electron polaron "miniband". All equivalent sites will have similar contribution to $K \propto P_{\nu}(1)$, adding to a constant factor.

[19] N. Zou, K. A. Chao, and Y. M. Galperin, Phys. Rev. Lett. 71, 1756(1993);

C. A. Stafford and N. S. Wingreen, Phys. Rev. lett., 76, 1916 (1996).

N. S. Wingreen, K. W. Jacobsen, and J. W. Wilkins, Phys. Rev. B 40, 11834 (1980).

[20] J. G. Chen, et al., Phys. Rev. B 43, 4531 (1991). 


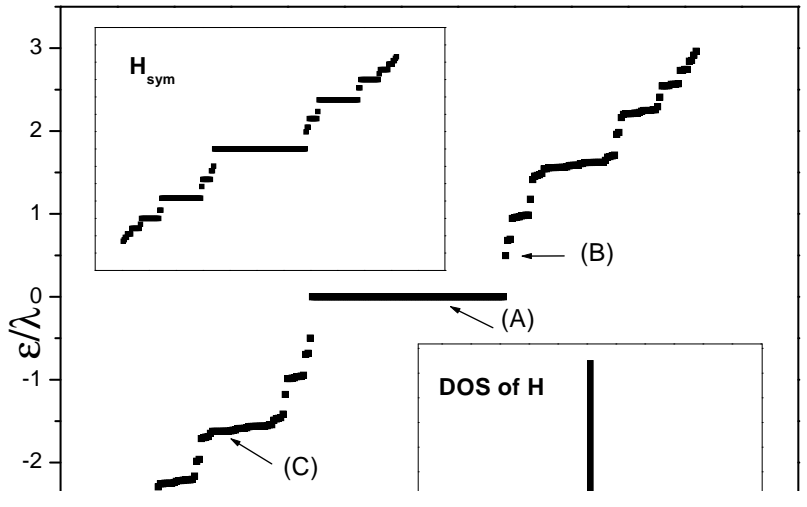

FIG. 1. Energy spectrum for $H$

$n=8$. Notice clear fractal structure of spectrum for $H_{\text {sym }}$, a metric about $\varepsilon=0$. Labels A to $\mathrm{C}$ relate to Fig. 2. Here, $\Delta=$

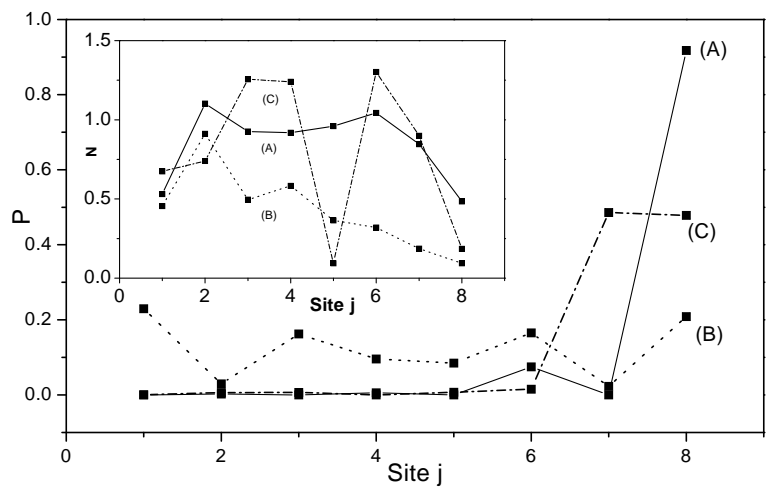

FIG. 2. Electronic amplitudes $P(j)$ at each site $j$ for several states, A to $\mathrm{C}$, as indicated in Fig. 1 . Notice A and C are localized near $j \simeq 8$. Panel shows phonon content $N(j)$ for same states. A and $\mathrm{C}$ have large phonon counts throughout and away from the electron, resulting in 'stretched' polarons.

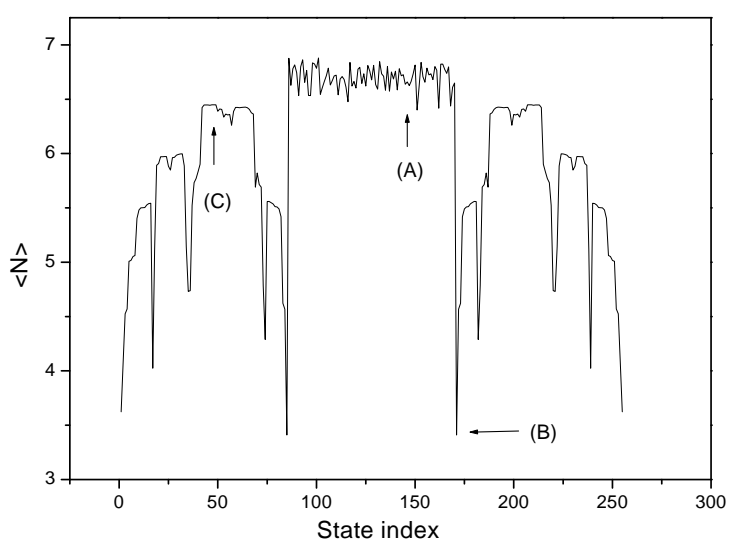

FIG. 3. Total phonon number for each eigenstate. Highest values plateau for the most localized electronic amplitudes, A and $\mathrm{C}$, while lowest counts correspond to extended electronic states, such as B. 


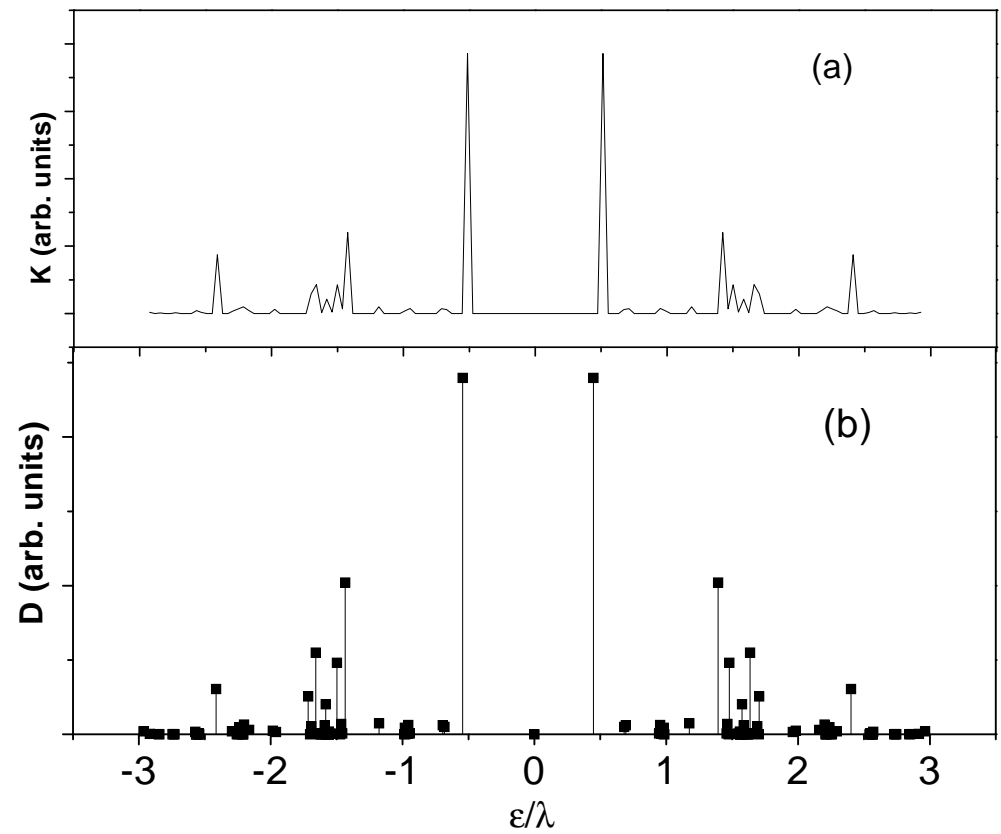

FIG. 4. (a) Optical absorption spectrum $K$ within miniband with level broadening added. (b) Tunneling amplitude $D$. Both cases for spectrum in Fig. 1. 